\title{
A Study of Detonation Interactions Inside a 2-D Ejector Using Detonation Transmission Tubing
}

\author{
H. Zare-Behtash, ${ }^{*}$ N. Gongora-Orozco, ${ }^{\dagger}$ and K. Kontis ${ }^{\ddagger}$ \\ School of MACE, The University of Manchester, M60 1QD, UK \\ G. Jagadeesh ${ }^{\S}$ \\ Indian Institute of Science, Bangalore 560 012, India
}

\begin{abstract}
The present experimental study employs a non-electrical (NONEL) tube placed inside a twodimensional convergent nozzle with an ejector system. The detonation initiates inside the NONEL tube, due to the reactants deposited on its internal surface, producing a shock wave and consequent products of combustion with repeatable results. High-speed shadowgraphy using the Shimadzu camera was employed to visualise the induced flow field. Pressure measurements were taken at different locations along the test section to study the flow quantitatively. The results revealed that the emergence of the products of combustion from the NONEL tube plays an important role in the flow dynamics within the ejector. The interaction between the combustion products and vortices formed due to shock diffraction lead to the early decay of the vortices. The presence of these vortices are vital in the entrainment of secondary flows through the ejector which is the main reason for the incorporation of ejectors in propulsion applications.
\end{abstract}

\footnotetext{
* Research Student, School of MACE, The University of Manchester, UK. AIAA Student Member ${ }^{\dagger}$ Research Student, School of MACE, The University of Manchester, UK.

${ }^{\ddagger}$ Professor (Reader), School of MACE, The University of Manchester, UK. AIAA Senior Member ${ }^{\S}$ Professor, Aerospace Engineering Department, IISc, India. AIAA Senior Member.
} 


\section{INTRODUCTION}

Studies into the diffraction of detonation waves have been motivated not only by the need to suppress accidental detonations but also in the interest of the applicability of such flows to the concept of pulse detonation engines (PDEs). Pulse detonation engines are currently being investigated as a new technology for aerospace propulsion. ${ }^{1-6}$ PDEs are unsteady propulsion devices that produce periodic impulse by utilising repetitive detonations. Ejectors are fluid pumps that are used to entrain secondary flows using a primary flow. For propulsion applications, this entrainment can augment thrust compared to that generated by the primary flow alone and thereby increase performance. ${ }^{7}$

For any given set of flight conditions there exists ejector inlet and outlet geometries which provide optimal performance. ${ }^{89}$ Similar to the findings of Kentfield, ${ }^{10}$ Etele et al. ${ }^{11}$ describe the ejector operation envelope as the low speed, low altitude phases of launch, where the entrainment and subsequent compression of the atmospheric air is largely responsible for any increased performance over traditional rockets. Non-detonational computational studies have highlighted the importance of the starting vortices, precursor shocks, and direct pressure loads created by the gas-dynamic processes within the ejector to the overall thrustaugmentation performance of the system. These data will be valuable for calibrating CFD codes and ultimately for the optimisation of PDE-ejector systems. ${ }^{12,13}$

Non-electrical (NONEL) tubes have been used extensively in blasting and mining, quarrying, crew escape systems in military aircraft, ordnance systems in launch vehicles and missiles which require the highest functional reliability. ${ }^{14}$ A NONEL DynoLine tube was used to generate the shock wave as result of detonation of the reactants inside the plastic tube. According the system description provided by Orica Mining Services, the velocity of the shock wave through the NONEL tube is approximately $2100 \mathrm{~m} / \mathrm{s}$. The present study examines both qualitatively and quantitatively the interaction of the resultant flow with a 2-D nozzle-ejector configuration. 


\section{EXPERIMENTAL SETUP}

\section{A. Nozzle-Ejector System}

The steel nozzle shown in Figure 1 has a uniform thickness, nominally $19.05 \mathrm{~mm}$, contraction ratio of $6: 1$, a throat height of $9.6 \mathrm{~mm}$, and a width of $10 \mathrm{~mm} .^{15}$ The ejector side walls were milled to shape and were bolted directly to the outer frame. The ejector side walls become parallel to the centre line at the plane of the nozzle exit with a distance of $45 \mathrm{~mm}$ between the upper and lower walls. The total length of the test section was $307 \mathrm{~mm}$ with a height of $209 \mathrm{~mm}$. Optical grade perspex sheets with a thickness of $10 \mathrm{~mm}$ were used on both sides of the nozzle to allow the visualisation of the flow.

Wall pressure measurements were conducted at the locations marked in Figure 1. The data was collected by means of a high-speed data acquisition system (National Instruments $P C I-6251)$. The initial pressure in the test section was ambient. The signal to begin pressure measurements and image acquisition, was obtained via a Kulite XT-190 transducer, mounted horizontally $5 \mathrm{~mm}$ from the exit of the NONEL tube, as shown in Figure 1.

\section{B. NONEL}

The flexible NONEL tube, with an outer diameter of $3 \mathrm{~mm}$ and internal diameter of $1 \mathrm{~mm}$, was inserted into an aluminium hypodermic tube with an internal diameter of $3.28 \mathrm{~mm}$. This would ensure that the tube would not be loose during firing and also allow sufficient room for easy changing of the NONEL tube after each run. $0.9 \mathrm{~m}$ of NONEL tubing was used for each run.

To initiate the detonation, a DynoStart 2 with a capacitance of $0.2 \mu F$ and output voltage of $2500 \mathrm{~V}$ was used. Both DynoLine and DynoStart were manufacture by Dyno Nobel Sweden AB (now, Orica Mining Services). The DynoLine contains $16 \mathrm{mg} / \mathrm{m}$ of Octogen (HMX) and $2 \mathrm{mg} / \mathrm{m}$ of Aluminium powder.

\section{High-Speed Shadowgraphy}

High-speed shadowgraphy ${ }^{16}$ with an optical arrangement similar to that used by Kontis et al., ${ }^{17}$ was employed to visualise the flow. The Shimadzu Hyper-Vision camera, capable of 
recording images at a rate of up to $1 \mathrm{Mfps}$, was used to create a motion picture of the flow field. Illumination for the Shimadzu camera was provided via a $300 \mathrm{~W}$ continuous Xenon lamp.

\section{RESULTS AND DISCUSSION}

The pressure measurement provided in Figure 2 is the measurement from the Kulite transducer used for triggering and is the average of three test runs. The repeatability of the experiment was calculated as $7 \%$.

Figure 3(a) shows the emergence of the shock front $(\mathrm{S})$ and the products of combustion (PC) from the NONEL tube placed at the entrance of the test section at the right hand edge of the image. The incident shock reflects from the walls of the test section, creating reflected shocks R1 and R2 in Figure 3(b). As the incident shock continues through the convergent portion of the nozzle and into the uniform area channel, multiple Mach waves (m) are visible underneath shocks R1 and R2 which are generated from the combustion cloud encased by the same shocks (Figure 3(c)). The hollow section, H, in the middle of the combustion cloud in Figure 3(c) forms due to the depletion of the products of combustion exiting the NONEL tube.

Shocks R1 and R2 approach each other head-on and cross over in Figure 3(d). At the same time the Mach waves appear to coalesce and become stronger $(\mathrm{M})$ due to the area reduction. Once shocks R1 and R2 have passed each other, they collide with the walls of the test section, marked in red in Figure 3(e); labelled R1' and R2' now, they move upstream towards the NONEL tube whilst the incident shock reached the end of the uniform area channel. The diffraction of the incident shock and the newly formed shock wave from the coalescence of the Mach waves(M in Figure 3(d)), generates two diffracted shocks DS1 and DS2 as well as two vortex cores (v), due the rolling of the shear layer between the emerging and ambient fluid in Figure 3(f).

Figure 4 shows the pressure traces of transducers T2 and T4 (see Figure 1 for locations). Transducer 2 shows two distinct peaks resulting from the passage of the incident shock S, and the products of combustion, PC. The following fluctuations are as a result of the Mach waves generated by the combustion cloud. The first peak S' of T4 is due to the arrival of the diffracted shock followed by a short duration pressure plateau. The pressure continues 
to increase (indicated by RS) when the diffracted shock reflects from the ejector walls and cross at the nozzle centreline. The pressure gradually reduces due to the expansion of the flow.

The diffracted shocks collide with the walls of the ejector and undergo regular reflection. As the effective wedge angle between the shock and the ejector reduces, the reflection type transitions to Mach reflection indicated by the presence of the Mach stem in Figure 5(a). In the same figure, the vortex cores (v) have travelled twice the height of the uniform area channel downstream, with the products of combustion (PC) arriving at the location of the cores. As the combustion products emerge from the nozzle they are drawn into the circulation of the two vortex cores (Figures 5(b) and 5(c)). Also, in Figure 5(b) we observe the formation of a separation region (SR) in the converging portion of the nozzle. The interaction between the vortex cores and products of combustions leads to the annihilation of the vortex cores. The products of combustion continue to move into the upper and lower atriums of the nozzle whilst at the same time moving further into the ejector segment (Figures 5(d)). The recirculation region continues to grow through Figures $5(\mathrm{c})$ to $5(\mathrm{~d})$.

The products of combustion fill the upper and lower atriums of the nozzle, as shown in Figure 6 by the red line. As the combustion products move further into the atriums (Figure 6(a)-6(b)), the pressure builds up. However, the compression in the atriums finally overcomes the momentum of the flow pushing the combustion products further in. This pushes the products of combustion back into the centre of nozzle, as seen by the reduced height of the red marker in Figure 6(c). The pressure trace of Figure 7 which is for the transducer located on the top right hand side (see Figure 1), shows the compression, A, and the consequent expansion of the flow, B.

\section{CONCLUSIONS}

Using a non-electrical (NONEL) tube with a combination of HMX and aluminium powder reactants deposited on the internal surface, a controlled and repeatable detonation was produced inside a 2-D nozzle-ejector system.

The incident shock wave generated within the NONEL tube travels through the test section unaffected by the products of combustion which emerge from the tube. The diffraction of this shock generates two vortex cores due to the rolling of the shear at the nozzle exit. 
A secondary shock wave forms from the coalescence and strengthening of the Mach waves, produced from the combustion cloud, moving through the converging nozzle. This led to a second diffracted shock wave.

The interaction between the products of combustion and the vortex cores leads to the demise of the vortices and the filling of the ejector section with combustion products. The combustion products continue to fill the test section cavities until the compression force built up overcomes the momentum of the flow and pushes the combustion products out from the cavities.

\section{Acknowledgments}

The authors are grateful to the technical and administrative staff at the school of MACE, and to the EPSRC engineering equipment loan pool for the loan of the Shimadzu system. 
1 Papalexandris, M.V., Thomas, J.F., Jacobs, C., Deledicque, V. "Structural characteristics of detonation expansion from a small channel to a larger one," Proceedings of the Combustion Institute, 2407-2414 (2007).

2 Ma, F., Choi, J.Y., Yang, V., "Thrust chamber dynamics and propulsive performance of singletube pulse detonation engines," Journal of Propulsion and Power 21, 512-526 (2005).

3 Oh, J.Y., Ma, F., Hsieh, S.Y., Yang, V., "Interactions between shock and acoustic waves in a supersonic inlet diffuser," Journal of Propulsion and Power 21, 486-495 (2005).

4 Ohyagi, S., Obara, T., Hoshi, S., Cai, P., Yoshihashi, T. "Diffraction and re-initiation of detonations behind a backward-facing step," Shock Waves 12, 221-226 (2002).

5 Wilson, J., Sgondea, A., Paxson, D.E., and Rosenthal, B.N., "Parametric investigation of thrust augmentation by ejectors on a pulsed detonation tube," Journal of Propulsion and Power 23, $108-115$ (2007).

${ }^{6}$ Glaser, A.J., Caldwell, N., Gutmark, E., Hoke, J., Bradley, R., Schauer, F. "Study on the operation of pulse-detonation engine-driven ejector," Journal of Propulsion and Power, 24, 1324-1331 (2008).

7 Etele, J., Parent, B., Sislian, J.P., "Analysis of increased compression through area constriction on ejector-rocket performance," Journal of Spacecraft and Rockets 44, 355-364 (2007).

8 Alperin, M., Wu, J.J., "Recent development of a jet-diffuser ejector," Journal of Aircraft 18, 1011-1017 (1981).

9 Alperin, M., Wu, J.J., "Thrust augmenting ejectors, part I," AIAA 21, 1428-1436 (1983).

10 Kentfield, J.A.C., "Fundamentals of idealised airbreathing pulse-detonation engines," Journal of Propulsion and Power 18, 77-83 (2002).

11 Etele, J., Sislian, J.P., Parent, B., "Effect of rocket exhaust configuration on ejector performance in RBCC engines," Journal of Propulsion and Power 21, 656-666 (2005).

12 Allgood, D., Gutmark, E., Rasheed, A., Dean, A.J. "Experimental investigation of a pulse detonation engine with a two-dimensional ejector," AIAA 43, 390-398 (2005).

13 Ma, F., Choi, J.-Y., Yang, V., "Propulsive performance of airbreathing pulse detonation engines," Journal of Propulsion and Power 22, 1188-1203 (2006).

14 Yang, L.C., Do, I.H.P., "Nonelectrical tube explosive transfer system," AIAA 38, 2260-2267 
(2000).

15 Eustace, V.A. "A study of two-dimensional supersonic air ejector systems," PhD Thesis, The University of Manchester Institute of Science and Technology (1969).

16 Settles, G.S. "Schlieren and Shadowgraph techniques," Springer Verlag (2001).

17 Kontis, K., An, R., Zare-Behtash, H., Kounadis, D., "Head-on collision of shock wave induced vortices with solid and perforated walls," Physics of Fluids 20 (2008). 


\section{List of Figures}

1 Test section. . . . . . . . . . . . . . . . . . . 10

2 Pressure trace of triggering transducer. . . . . . . . . . . . . . . 10

3 Segment I of flow analysis. . . . . . . . . . . . . . . . . . . . 11

4 Pressure history of transducers T2 and T4 (see Figure 1) . . . . . . . . 11

5 Segment II of flow analysis. . . . . . . . . . . . . . . . . . . 12

6 Segment III of flow analysis. . . . . . . . . . . . . . . . . . . . . . . 13

$7 \quad$ Pressure trace of transducer T5 . . . . . . . . . . . . . . . . . . 13 


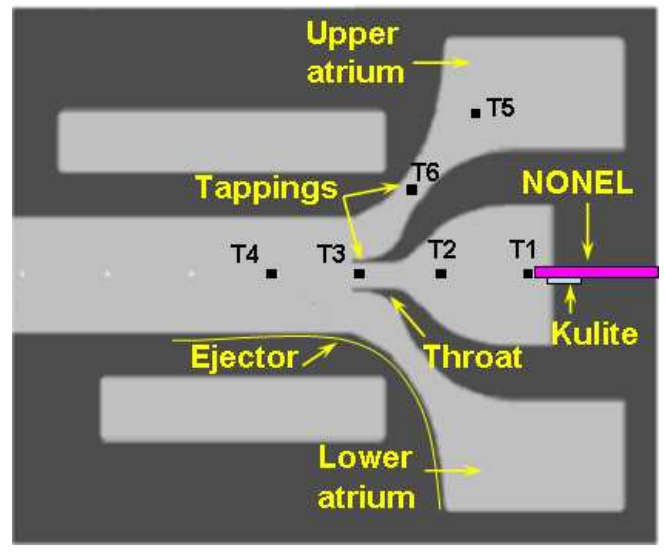

FIG. 1: Test section.

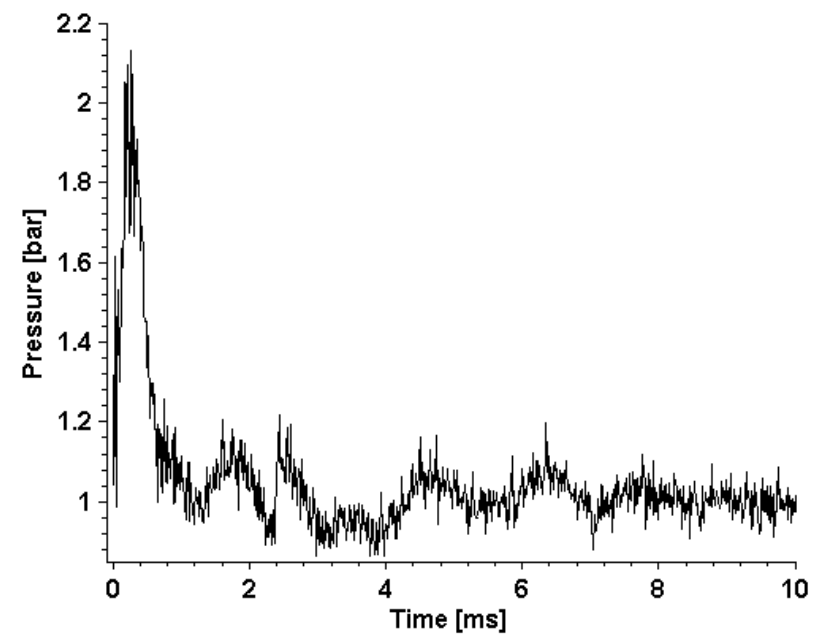

FIG. 2: Pressure trace of triggering transducer. 


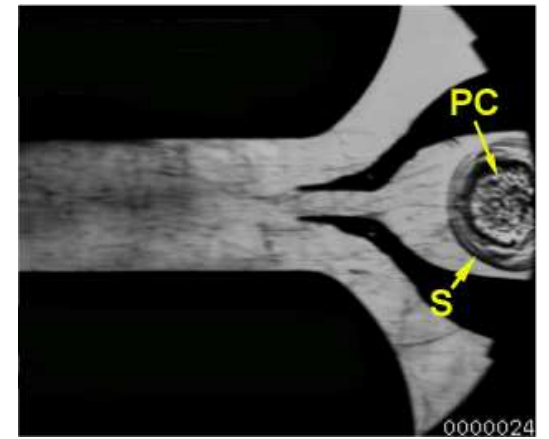

(a)

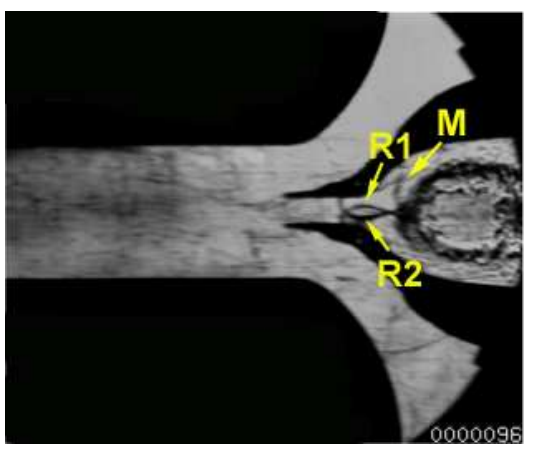

(d)

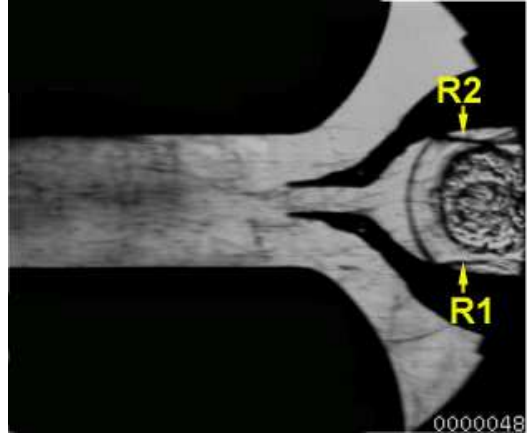

(b)

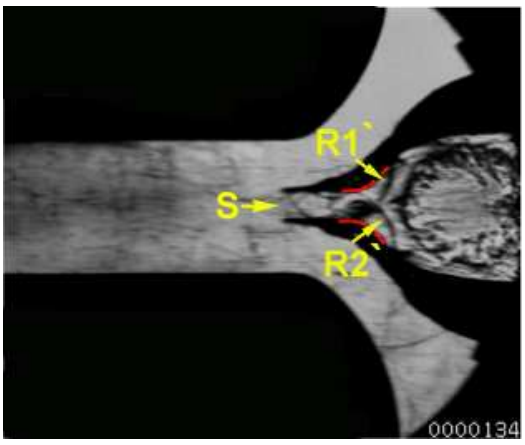

(e)

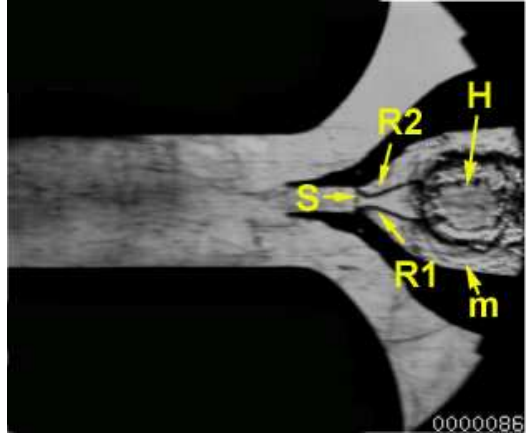

(c)

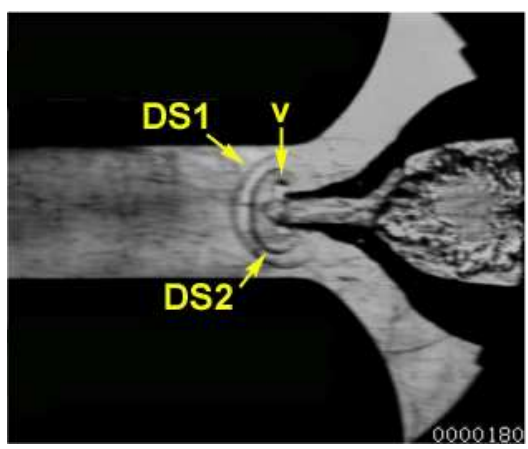

(f)

FIG. 3: Segment I of flow analysis.

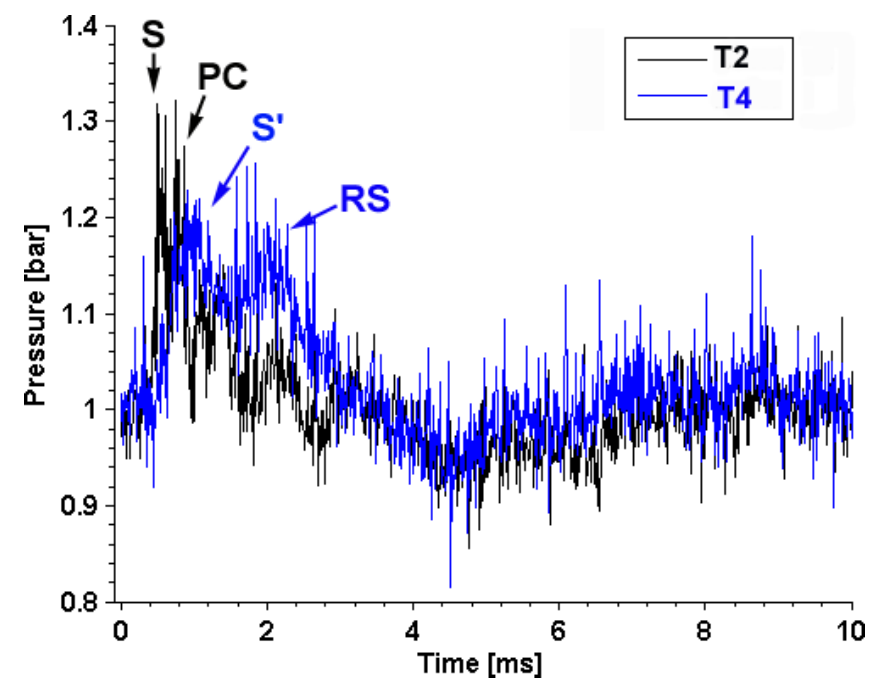

FIG. 4: Pressure history of transducers T2 and T4 (see Figure 1). 

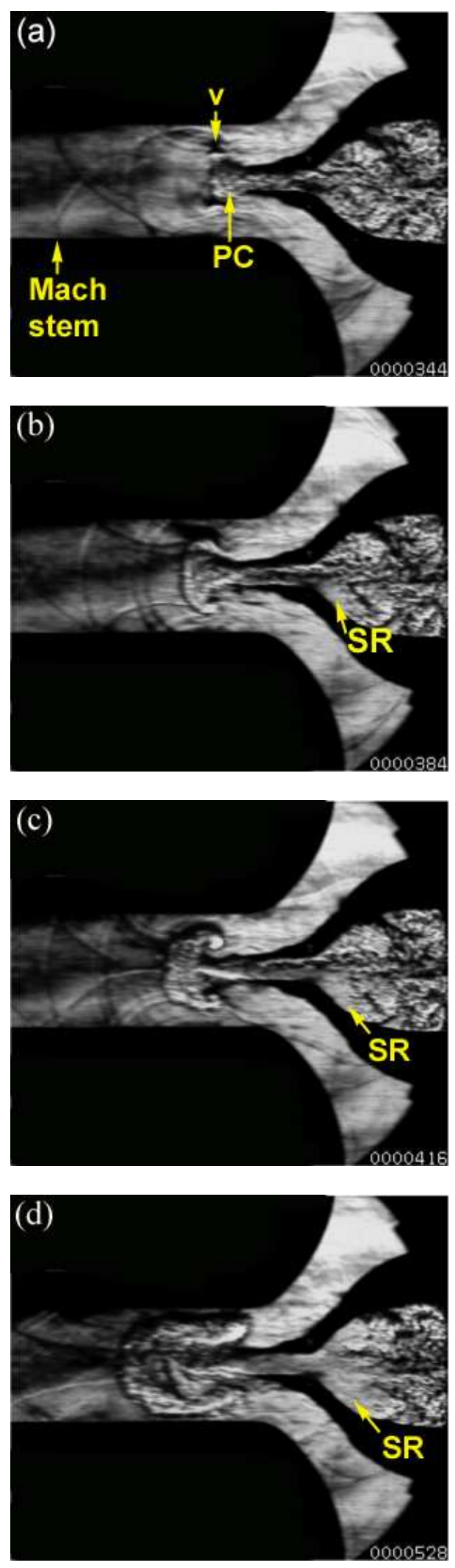

FIG. 5: Segment II of flow analysis. 


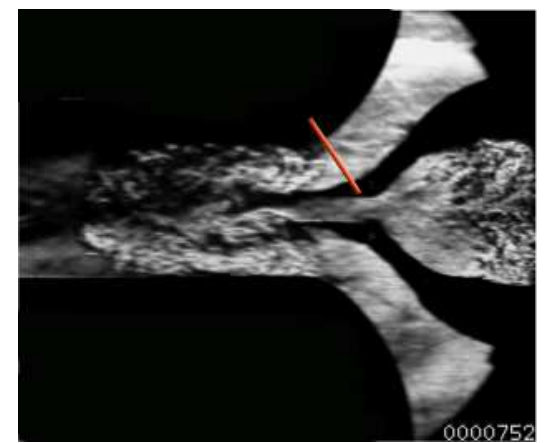

(a)

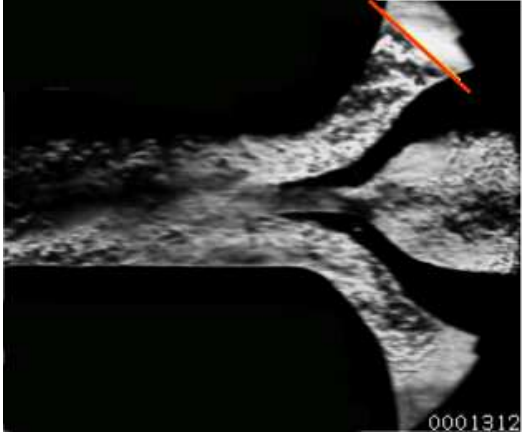

(b)

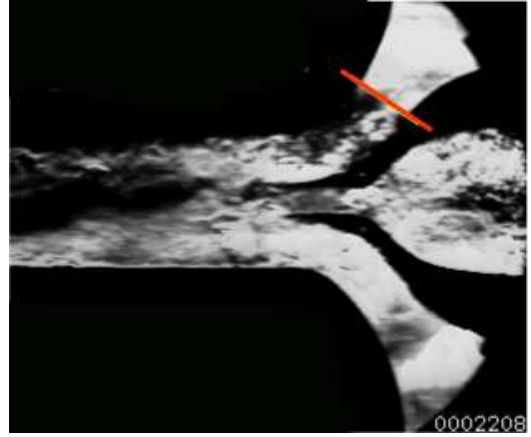

(c)

FIG. 6: Segment III of flow analysis.

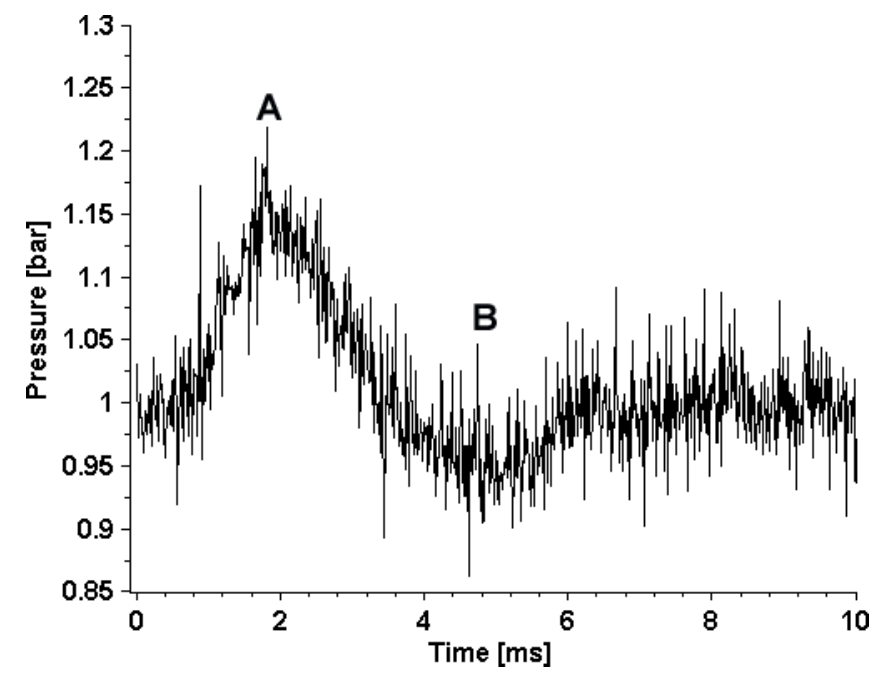

FIG. 7: Pressure trace of transducer T5. 